\title{
VOXEL TREE MODELING FOR ESTIMATING LEAF AREA DENSITY AND WOODY MATERIAL VOLUME USING 3-D LIDAR DATA
}

\author{
F. Hosoi, Y. Nakai, K.Omasa \\ Graduate School of Agricultural and Life Sciences, The University of Tokyo, Yayoi 1-1-1, Bunkyo-ku, \\ Tokyo 113-8657, Japan- ahosoi@mail.ecc.u-tokyo.ac.jp
}

KEY WORDS: Broad leaf, Leaf area density, Lidar, Point cloud, 3D, Voxel model, Woody material volume

\begin{abstract}
:
In this work, the main focus is on voxel tree modeling using 3-D lidar data for accurate leaf area density (LAD) and woody material volume estimation. For more accurate LAD estimation, the voxel model was constructed by combining airborne and portable ground-based lidar data. The profiles obtained by the two types of lidar complemented each other, thus eliminating blind regions and yielding more accurate LAD profiles than could be obtained by using each type of lidar alone. Parts of the LAD profiles that were underestimated even when data from both lidars were combined were interpolated by using a Gaussian function, yielding improved results. A laser beam coverage index, $\Omega$, incorporating the lidar's laser beam settings and a laser beam attenuation factor, was proposed. This index showed general applicability to explain the LAD estimation error for LAD measurements using different types of lidars. In addition, we proposed a method for accurate woody material volume estimation based on a 3-D voxel-based solid modeling of the tree from portable scanning lidar data. The solid model was composed of consecutive voxels that filled the outer surface and the interior of the stem and large branches. By using the model, the woody material volume of not only the whole target tree but also of any part of the target tree can be directly calculated easily and accurately by counting the number of corresponding voxels and multiplying the result by the per-voxel volume.
\end{abstract}

\section{INTRODUCTION}

Trees have important functional roles, including the cycling of materials and energy through photosynthesis and transpiration, the maintenance of microclimates, and the provision of habitats for various species. To understand the relationship between these functions and structure, many structural parameters, including height, stem diameter, leaf area density, volume, and biomass, have been measured by a variety of methods.

Recently, lidar (light detection and ranging) has been applied to tree structural measurements (Hyyppä et al, 2001; Lefsky and McHale, 2008; Popescu et al., 2003; Næsset et al., 2004; Hosoi and Omasa, 2006, 2007, Hosoi et al., 2010, 2013). Portable scanning lidar systems can capture the complex shape and structure of individual trees as a detailed 3-D point-cloud image on the ground. Thus, 3-D tree models faithfully reproduced from the lidar-derived 3-D point-cloud image can be used to estimate tree structural parameters. Several 3-D tree modeling techniques have been employed for the estimation of individual tree structural parameters from portable scanning lidar data (Dassot et al., 2011). An approach to 3-D tree modeling based on lidar-derived point-cloud data is voxel representation (Côté et al., 2011; Hosoi and Omasa, 2006; Lefsky and McHale, 2008; Schilling et al., 2012). Hosoi and Omasa (2006) recently proposed a voxel-based method of 3-D modeling that uses portable scanning lidar data (Voxel-based Canopy Profiling method). In this method, lidar data points are converted into voxel elements in a 3-D voxel array to faithfully reproduce the canopy as a voxel model. This method has been used for estimating vertical leaf area density (LAD) profiles, offering the accurate estimates when sufficient numbers of laser beams were supplied to the canopy. However, the ground-level measurements often showed a tendency to underestimate the upper canopy, when the laser beams are obstructed by the middle and lower parts of the target canopy itself. Such underestimation should be improved. The improvement requires the appropriate determination of the measurement configuration (e.g. laser beam settings, lidar position) according to the canopy structure. Thus, a criterion to determine the appropriate configuration is needed, but such a criterion has not yet been proposed. In addition, although voxel modelling can be used to estimate other structural parameters besides LAD, the potential has not yet been fully enhanced for retrieving other important structural parameters such as woody material volume.

Based on the above, we propose a method to improve the accuracy of LAD estimation of a canopy based on 3-D voxel tree modeling by compositing airborne and portable scanning lidar systems. In addition, we propose a criterion to explain the LAD estimation error and to be usable for determining appropriate lider configurations. Moreover, a method to estimate woody material volume from a lidar-derived 3-D voxel tree model is proposed.

\section{METHODS}

\subsection{3-D data acquisition and registration}

The target trees are scanned from several measurement points surrounding them and laser beams are optimally inclined to facilitate full laser beam illumination of whole target up to the internal. Lidar data obtained in leafy and leafless conditions are respectively used for estimating $\mathrm{LAD}$ profiles and woody material volume. The complete data set is composed of several point cloud data, one obtained from each of the several measurement positions. These data with their individual coordinate systems are registered into a single-point cloud data set with a common coordinate system. 


\subsection{Voxelization}

All points within the registered data are converted into voxel coordinates. All points within the registered data are converted into voxel coordinates by the following equations.

$i=\operatorname{Int}\left(\frac{X-X_{\min }}{\Delta i}\right)+1, j=\operatorname{Int}\left(\frac{Y-Y_{\min }}{\Delta j}\right)+1, k=\operatorname{Int}\left(\frac{Z-Z_{\min }}{\Delta k}\right)+1$

where $(i, j, k)$ represents the voxel's coordinates within the voxel array, Int is a function that rounds off the result of the calculation to the nearest integer, $(X, Y, Z)$ represents the point coordinates of the registered lidar data, $\left(X_{\min }, Y_{\min }, Z_{\min }\right)$ represent the minimum values of $(X, Y, Z)$ and $(\Delta i, \Delta j, \Delta k)$ represent the voxel element size. Voxels corresponding to coordinates converted from points within the registered data are assigned 1 as the attribute value. A voxel with the attribute value of 1 represents a voxel in which at least one laser beam is intercepted by leaves or woody material. For retrieving wood material volume, these voxels with the attribute value of 1 are used for the subsequent processes (details are explained in 2.4). On the other hand, for retrieving LAD, additional voxels that are assigned as the other attribute value are required for the computation. For this reason, all laser beams emitted from the lidar positions are then traced within the voxel array in accordance with the actual laser beam angles. If voxels that do not have an attribute value of 1 are intersected by at least one laser beam trace, the voxel was assigned 2 as the attribute value. A voxel with an attribute value of 2 therefore represents a voxel through which one or more laser beams passed without touching a leaf.

\subsection{Derivation of LAD and laser beam coverage index}

\subsubsection{LAD computation}

Based on the attribute values of 1 and 2, LAD is computed in each horizontal layer of the canopy using the following equation:

$$
\operatorname{LAD}(h, \Delta H)=\frac{\cos \theta}{G(\theta)} \cdot \frac{1}{\Delta H} \sum_{k=m_{h}}^{m_{h+\Delta H}} \frac{n_{\mathrm{I}}(k)}{n_{\mathrm{I}}(k)+n_{\mathrm{p}}(k)}
$$

where $\theta$ is the zenith angle of a laser beam, $\Delta H$ is the horizontal layer thickness, and $m_{h}$ and $m_{h+\Delta H}$ are the voxel coordinates on the vertical axis equivalent to height $h$ and $h+\Delta H$ in orthogonal coordinates $\left(h=\Delta k \times m_{h}\right) . n_{\mathrm{I}}(k)$ and $n_{\mathrm{P}}(k)$ are the numbers of voxels with the attribute values of 1 and 2 in the $k$ th horyzontal layer of the voxel array, respectively. Thus, $n_{\mathrm{I}}(k)+n_{\mathrm{P}}(k)$ represent the total number of incident laser beams that reach the $k$ th layer and $n_{\mathrm{I}}(k) /\left(n_{\mathrm{I}}(k)+n_{\mathrm{P}}(k)\right)$ represents contact frequency of laser beams on the canopy. $G(\theta)$ is the mean projection of a unit leaf area on a plane perpendicular to the direction of the laser beam at $\theta$ ). The term $\cos (\theta)[G(\theta)]^{-1}$ is a correction factor for the influence of leaf inclination angle and laser beam direction. $\cos (\theta)[G(\theta)]^{-1}$ is determined using the distribution of leaf inclination angles.

\subsubsection{The laser beam coverage index}

The laser beam coverage index $\Omega$ is an indicator of the proportion of the area of a horizontal plane within the canopy covered by laser beams. The index can be used as a criterion to estimate accuracy of lidar-derived LAD estimation and to appropriately determine laser beam settings. Three factors that relate to the coverage are selected: the horizontally projected area of the laser beam $\left(A_{\text {beam }}\right)$, the number of incident laser beams per unit area of a horizontal plane $(N)$, and a laser beam attenuation factor $\left(K \cdot L A I_{\text {cum }}\right)$. In the laser beam attenuation factor, $K$ is a parameter for the influence of the leaf inclination angle and the laser beam direction on laser beam attenuation and is equivalent to the inverse of the correction factor $\cos (\theta)[G(\theta)]^{-1}$ described in eq.(2). $L A I_{\text {cum }}$ is the lidar-derived cumulative LAI at a certain height. $K$ and $L A I_{\text {cum }}$ reflect the structural attributes of the canopy, while $A_{\text {beam }}$ and $N$ relate to the laser beam settings. The laser beam coverage index $\Omega$ is made in relation to these three factors as follows:

$$
\Omega=A_{\text {beam }} \times N \exp \left(-K \cdot L A I_{\text {cum }}\right)
$$

The index $\Omega$ is defined as the total horizontally projected area of the incident laser beams per unit area of a horizontal plane within the canopy at certain height. This is also translated as the proportion of the area of a horizontal plane within the canopy covered by laser beams at certain height. $A_{\text {beam }}$ and $N$ are determined from the laser beam settings. $K \cdot L A I_{\text {cum }}$ is calculated for each height by using the voxel attributes, as follows:

$$
K \cdot L A I_{\text {cum }}=\sum_{k=m_{\text {botom }}}^{m_{h}} \frac{n_{I}(k)}{n_{I}(k)+n_{p}(k)}
$$

where $n_{I}(\mathrm{k})$ is the numbers of voxels at which laser beams are intercepted and $n_{P}(\mathrm{k})$ is the ones through which laser beams passed. $m_{h}$, and $m_{\text {bottom }}$ are the voxel coordinates on the vertical axis equivalent to height $h$ and the heights of the canopy bottom. Eq.(4) is for ground-based portable lidar measurements, but it can be used for airborne lidar measurements, by replacing $m_{h}$ with $m_{\text {top }}$ (equivalent to height of the canopy top) and $m_{\text {bottom }}$ with $m_{h}$.

\subsection{Derivation of woody material volume}

For estimating woody material volume, a voxel solid model of the entire tree is produced and used, at which consecutive voxels fill the outer surface and the interior of the stem and large branches. Although this is categorized as voxel tree modelling, the procedures are different from the ones used in LAD estimation. The procedures of the model production and volume estimation are the following three steps.

\subsubsection{Separating the stem and large branches from small branches}

After the initial voxelization of the target (section 2.2), the voxels of the stem and larger branches is separated from those of the small branches. First, several voxels corresponding to the stem or large branches are manually picked up from the voxelized 3-D model. These are used as starting points for separating the stem and large branches from small branches. Then, voxels neighbouring these initial voxels within a certain distance are categorized as stem or large-branch voxels. The categorized voxels then become new initial (starting) points and the above searching procedure is repeated. This procedure is iterated until no more voxels can be categorized as stem or large branches. Then, all of the remaining voxels are categorized as small-branch voxels (see Hosoi et al., 2013 for the details).

\subsubsection{Surface generation}

After the above separation, a surface generation procedure is applied. The surface generation procedure interpolates additional voxels between the initial voxels of each one-voxelthick horizontal layer of the voxelized model to obtain a continuous closed curve representing the exterior surface of the 
stem and each large branch.

To generate the surfaces, the voxels within a horizontal layer are classified into sets, each one corresponding to the stem or to a large branch. To do this, a grid is first superimposed on each horizontal voxel layer. The grid size is chosen to be larger than both the original voxels and the laser beam pitch (Fig. 1B). Then, each cell of the grid that included at least one voxel is filled (labeled) and Moore's neighbor-tracing algorithm is used to connect adjacent filled cells to trace the approximate contour of the exterior surface of the stem or large branch (Ghuneim, 2009). In this method, cells in each row of the grid, starting from the top row and proceeding to the bottom row, are scanned from the leftmost to the rightmost. Then, the first-encountered filled cell is treated as a starting cell and the eight neighbouring cells are examined in a clockwise direction until another filled cell is encountered; this encountered cell is then treated as the next cell in the contour. These steps are repeated until the starting cell is again encountered. This procedure yields a closed curve outlining the stem or a large branch. During this procedure, the starting cell and each selected cell are labeled (a certain value is given to each cell as its attribute value), thus producing a set of labeled cells corresponding to the stem or to a large branch. Next, scanning of the grid is repeated, skipping already labeled cells, and the first-encountered filled and unlabeled cell is treated as the next starting cell. The same procedure is applied to the other filled cells, with different labels being used to discriminate the stem and each large branch (Fig. 1B: (a), (b), and (c)). In this way, the voxels included in the cells are classified into sets, each of which corresponded either to the stem or to a specific large branch.

Next, a contour interpolation procedure is applied to each set of classified voxels (Fig. 1C) within a horizontal layer. Here, the superimposed grid cells described above are no longer used. To generate contours of the exterior surfaces of the stem and each large branch composed of consecutive voxels, gaps (empty voxels) between voxels in a voxel set are filled by linear interpolation. First, voxels that should not be included in a contour are excluded as noise (Fig. 1C). To judge whether a voxel is noise, the mean $\left(d_{\mathrm{m}}\right)$ and standard deviation $(\sigma)$ of the distance between each voxel and the centroid of the voxel set is calculated. Voxels at a distance of more than $d_{\mathrm{m}}+3 \sigma$ are regarded as noise and excluded them from the set. Next, The angle between the $x$-axis and each line segment connecting a voxel with the centroid of the voxel set is calculated. The angles are then sorted in ascending order to determine the tracking order of each voxel. Then, to generate the contour, a certain tracked voxel are picked and then the empty voxels between that voxel and the next filled voxel are filled in the tracking order by linear interpolation (Fig. 1C). This procedure is repeated until all voxels composing the contour of the exterior surface of the stem or large branch have been filled (that is, until a continuous contour composed of consecutively filled voxels have been generated). This procedure is applied to each classified voxel set within a horizontal voxel layer and repeated from the lowest to the highest horizontal layer, thus generating exterior surfaces of the stem and each large branch composed of consecutive voxels.

\subsubsection{Internal filling and volume estimation}

The interiors of the stem and each large branch are filled by scanning between the voxels of a contour in both the $x$ and $y$ directions (Fig. 1D). For instance, for a scan in the $x$ direction, a starting voxel near the lower left of the contour is picked and a line is traced in the positive $x$ direction from the starting voxel to the voxel on the opposite side of the contour. The first voxel is equivalent to the minimum and the last voxel to the maximum $\mathrm{x}$-coordinate value along the scan line, and all empty voxels between the starting and ending voxels are labeled. This procedure is repeated for each voxel along the contour in both the $x$ and $y$ directions. Then, those voxels that have been labeled twice (that is, by both the $x$ - and $y$-direction scans) are regarded as interior voxels of that contour and filled them. This interior filling procedure is applied to the stem and all large branches within each horizontal voxel layer, from the lowest to the highest horizontal layer. In this way, both the surfaces and interiors of the entire stem and all large branches are filled with consecutive voxels. Then a $3-D$ voxel-based solid model of the whole target tree is produced by merging the filled stem and large branches with the voxels previously categorized into small branches (Sec. 2.4.1).
(A)

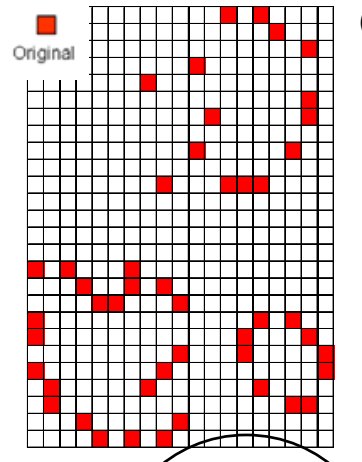

(C)

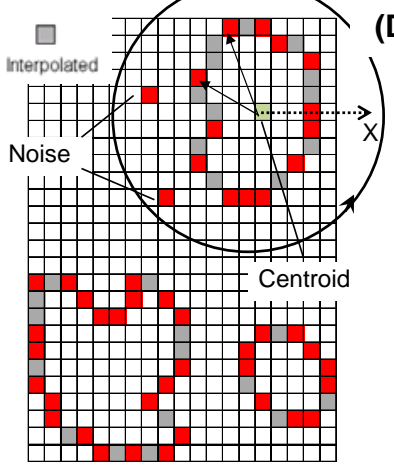

(B)

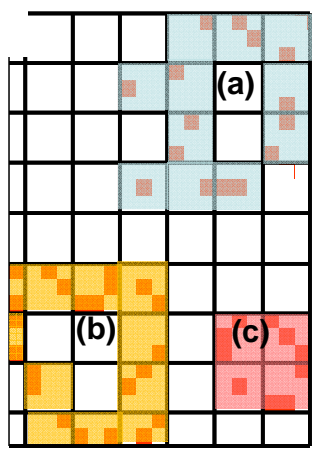

(D)

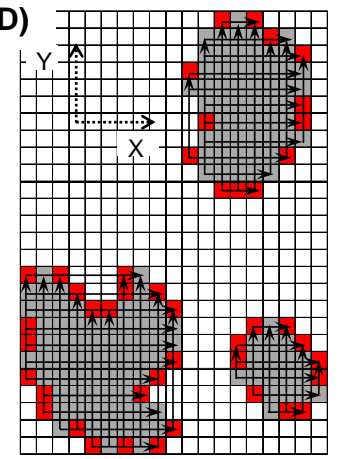

Figure 1: Generation of the exterior surface contours of the stem and large branches. (A) Original voxels within a horizontal layer obtained by conversion of the lidar data points. (B) A grid was overlaid on a one-voxel-thick horizontal layer, and voxel sets (a), (b), and (c), corresponding to the contours of the stem and each large branch are identified by connecting cells using a neighbor-tracing algorithm. (C) Contours of the stem and of large branches are filled by interpolating voxels (light gray) between the original voxels. (D) Interior voxels are identified and filled by scanning in the $x$ and $y$ directions from voxels on the contour (Hosoi et al., 2013).

The volume of the woody material is calculated from the generated 3-D voxel-based solid model. This model is composed of consecutive voxels that filled the outer surfaces and the interiors of the stem and large branches, and a cloud of voxels equivalent to small branches that are discretely scattered in mainly the upper part of the target. By using the model, we can easily estimate the woody material volume of any part of the tree by counting the number of voxels within the part and then multiplying the number of voxels by the unit voxel volume. 


\section{EXPERIMENTS}

\subsection{LAD estimation for broad-leaved woody canopy using a portable and airborne lidar}

The experiment was conducted in a mixed plantation in Ibaraki Prefecture, $40 \mathrm{~km}$ northeast of central Metropolitan Tokyo, Japan (Hosoi et al., 2010). The topography was nearly flat. A $4 \times 8$-m measurement plot was established at the site (Fig. 2 (A)), and the Japanese zelkova canopy within the plot was used for the experiment. The plot was divided into eight $2 \times 2-\mathrm{m}$ quadrats (Fig. 2 (B)) and each vertical region within each of the quadrats was divided into 16 cells (each $2 \times 2 \times 0.5 \mathrm{~m}$ ) between heights of 5 to $13 \mathrm{~m}$ above the ground. The entire canopy within the measurement plot was thus divided into 128 cells. The actual LAD in each cell was measured directly by stratified clipping in September 2005, the month following the lidar measurements of the leafy canopy.
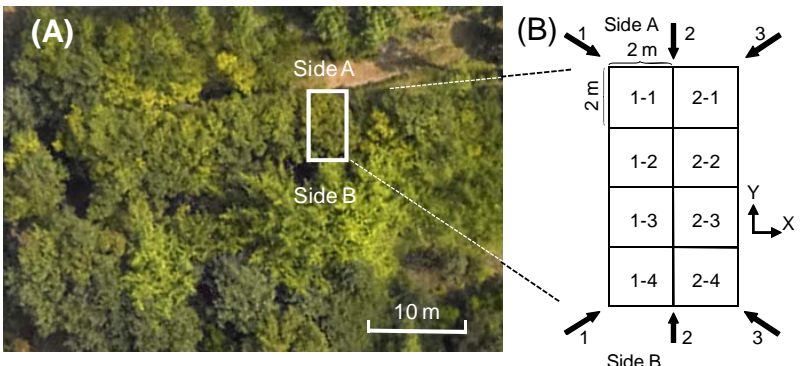

Figure 2: Study area. (A): Aerial photograph (B): A measurement plot established beneath the zelkova canopy. Arrows in (b) show the directions in which lidar scanning was performed (modified from Hosoi and Omasa, 2007).

In this experiment, not only ground-based portable scanning lidar but also airborne lidar was used for LAD estimation because the combination of airborne and portable ground-based scanning lidar could potentially allow accurate estimation of LAD by eliminating the blind regions of each lidar. Measurements using portable ground-based scanning lidar (LPM-25HA, RIEGL, Austria; the range accuracy of $\pm 8 \mathrm{~mm}$ ) were conducted in August 2005 as reported in (Hosoi et al., 2010). The measurement plot and the area around the plot were also scanned in August 2005 from above by a lidar mounted on a helicopter (ALTM 3100 DC, Optech Co., and Aero Asahi Co., Japan). The range accuracy and horizontal accuracy were within 15 and $13 \mathrm{~cm}$, respectively. The footprint interval, i.e. the distance between centers of adjacent laser beams on the ground, was $0.29 \mathrm{~m}$ in the direction of the scan and $0.26 \mathrm{~m}$ in the direction of flight.

3-D point cloud data obtained by airborne and portable groundbased lidars were registered into a common coordinate system as shown in Fig.3. To facilitate the registration between the data taken by different lidar systems, houses neighbouring the study area were scanned by the two lidars and used as references for the registration. Figure 4 shows the LAD profiles for the entire measurement plot, obtained from actual measurements and from the two lidars. The portable ground-based lidar data and the actual ones were cited from (Hosoi and Omasa 2007). LAD estimates from the airborne and portable ground-based lidar data agreed well with the actual values for the upper and lower canopy, respectively, but underestimated the values at around $10 \mathrm{~m}$ height. Thus, the profile needed to be interpolated with a
Gaussian function. As a result, LAD profile was improved by the interpolation with the MAE (Mean Absolute Error) of 0.13 $\mathrm{m}^{2} \mathrm{~m}^{-3}$. Although a portable scanning lidar used in this experiment (LPM-25HA) was an old model, up-to-date lidar systems that record multiple returns or full-waveforms can get more information about canopy inside. The use of such instruments would improve the accuracy of the present method.

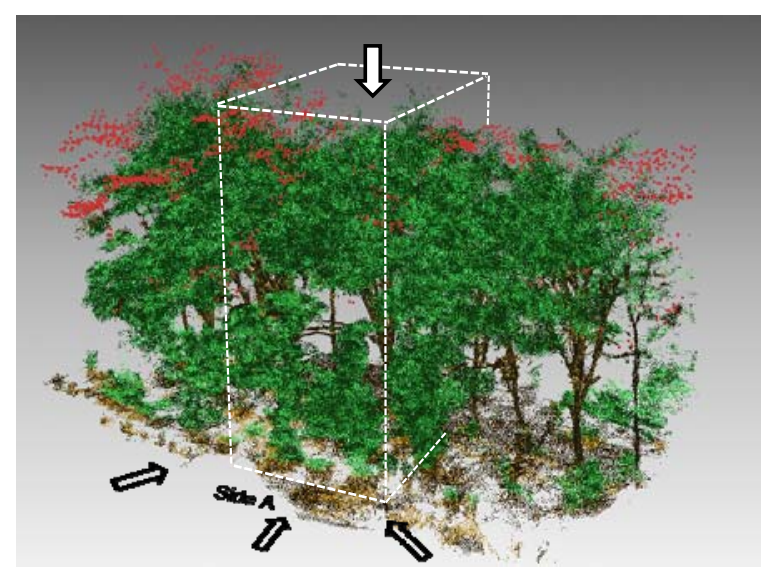

Figure 3: A 3-D image of the Japanese zelkova canopy at the study site, obtained by registration of the images measured from above with the airborne lidar and from the six ground positions with portable ground-based lidar (the data obtained from the airborne lidar are colored red: Hosoi et al., 2010) .

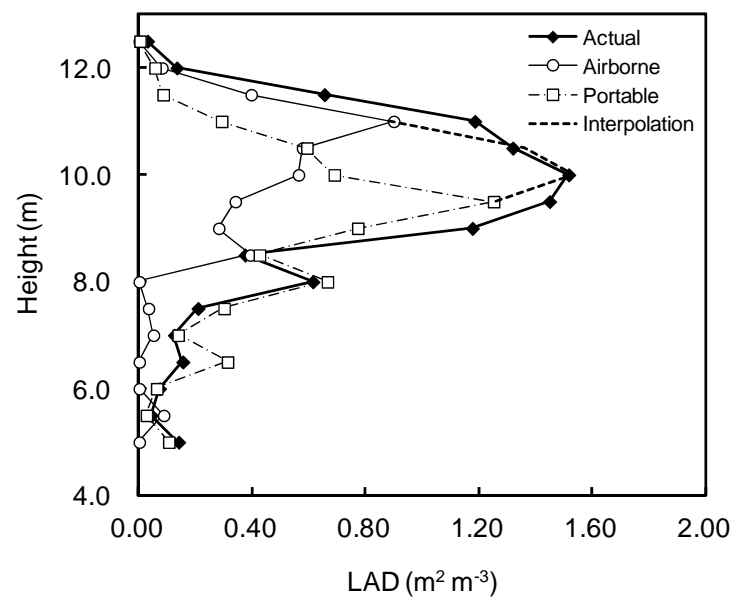

Figure 4: Comparison of LAD profiles for the measurement plot estimated from airborne and portable ground-based lidar measurements and the actual LAD profile (modified form Hosoi et al., 2010).

The relationships between the laser beam coverage index, $\Omega$ (Eq. 3), and the absolute error of the LAD estimates are shown in Fig. 5. In spite of the different laser beam settings of the two lidars, the points from both lidars in the scatter plot were found in the same general region on the plot, indicating that the relationship between the index $\Omega$ and the LAD estimation error was similar between the two lidars. The absolute errors of both lidars began to increase drastically when $\Omega$ decreased to around 1.0. Large errors were associated with $\Omega$ less than or equal to approximately 1.0 , whereas smaller errors were associated with values of $\Omega$ increased above 2.0. When the value of $\Omega$ exceeds 1.0 , the laser beams can illuminate the entire horizontal plane, but when it is less than 1.0, then some parts of the horizontal 
plane cannot be illuminated by the laser beams. In the former case, the LAD error decreases because the number of laser beams incident into the canopy is sufficient, but in the latter case, the error increases due to a lack of information about the canopy caused by an insufficient number of laser beams incident into the canopy. This explains the results shown in Fig.5, in which the LAD error began to increase drastically at values of $\Omega$ near 1.0. Thus, for better LAD estimation, the value of $\Omega$ should exceed 1.0. In practice, the value of $\Omega$ should be more than 2.0 to be certain of obtaining accurate results. Therefore, in practical lidar measurements, the measurement settings such as the laser beam settings and the lidar positions should be chosen such that $\Omega$ becomes as large as possible exceeding 2.0. In addition, it is notable that a similar tendency was observed in the relationship between $\Omega$ and LAD error for both airborne and ground-based lidars in spite of their different setting values for $A_{\text {beam }}$ and $N$, as shown in Fig. 4. This means that $\Omega$ can be used to assess the LAD error of lidar measurements made by using different settings and also suggests that $\Omega$, that is, the proportion of the area covered by laser beams in a horizontal plane, is an essential and practical factor that relates to estimation accuracy in lidar-based LAD measurements.

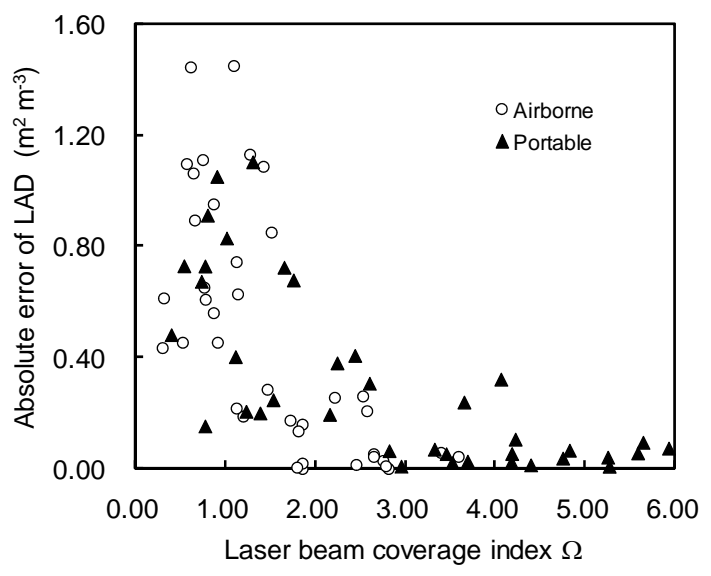

Figure 5: Relationship between the laser beam coverage index $\Omega\left(=A_{\text {beam }} \times N \exp \left(-K L A I_{\text {cum }}\right) ;\right.$ Eq. 3$)$ and the absolute errors of LAD in all airborne and portable ground-based lidar measurements (Hosoi et al., 2010).

\subsection{Volume estimation for a broad-leaved tree}

This experiment was conducted in January 2010, during leaf-off conditions, with a portable ground-based scanning lidar (Hosoi et al., 2013). The measurement site, portable scanning lidar system and lidar-set-up were similar to the ones explained in 3.1. Fig.6 B shows the 3-D voxel-based solid model of the zelkova tree generated from the 3 -D point cloud data obtained by the portable scanning lidar system. The voxel size was 0.5 $\mathrm{cm} \times 0.5 \mathrm{~cm} \times 0.5 \mathrm{~cm}$. The small branches were well separated from the stem and large branches, and a cross-sectional view of the stem shows that both the outer surface of the model and its interior are composed of consecutively filled voxels (Fig. 6C).

Examination of the estimated volume at each height (Fig. 7) showed that the volume of the stem and large branches increased as the height decreased, reaching its maximum in the lowest height interval (Fig.7). Conversely, the volume of the small branches increased with height and reached its maximum value at $12.00 \mathrm{~m}$. As a result, between heights of 0.00 and 9.00 $\mathrm{m}$, most of the total volume was accounted for by the stem and large branches, but above $9.00 \mathrm{~m}$ height, most of the volume was in small branches. In the whole target, the volume of the stem and large branches was $0.417 \mathrm{~m}^{3}$, and that of the small branches was $0.135 \mathrm{~m}^{3}$, for a total volume of $0.552 \mathrm{~m}^{3}$ for the whole target.

Direct stem and branch measurements and model-estimated stem and branch volume demonstrated strong agreement (Fig. 8 ). The mean absolute percentage error, obtained by calculating the absolute percentage error in each height interval and then averaging them, was $6.8 \%$. The error of the total volume of the part, obtained by summing the volumes of each height interval, was $0.5 \%$. The estimated percentage error corresponding to the sampled part of the small branches, obtained by comparing the estimated volume with the directly measured value, was $34.0 \%$. One advantage of this volume estimation method compared with other methods that use allometric equations or approximations of stem and branch shapes is that no allometric equations are needed and shapes are not approximated, so that the complex shaped tree volume can be estimated accurately. Another advantage is that by using the model, the woody material volume of not only the whole target tree but also of any part of the target tree can be directly calculated easily and accurately by counting the number of corresponding voxels and multiplying the result by the per-voxel volume.

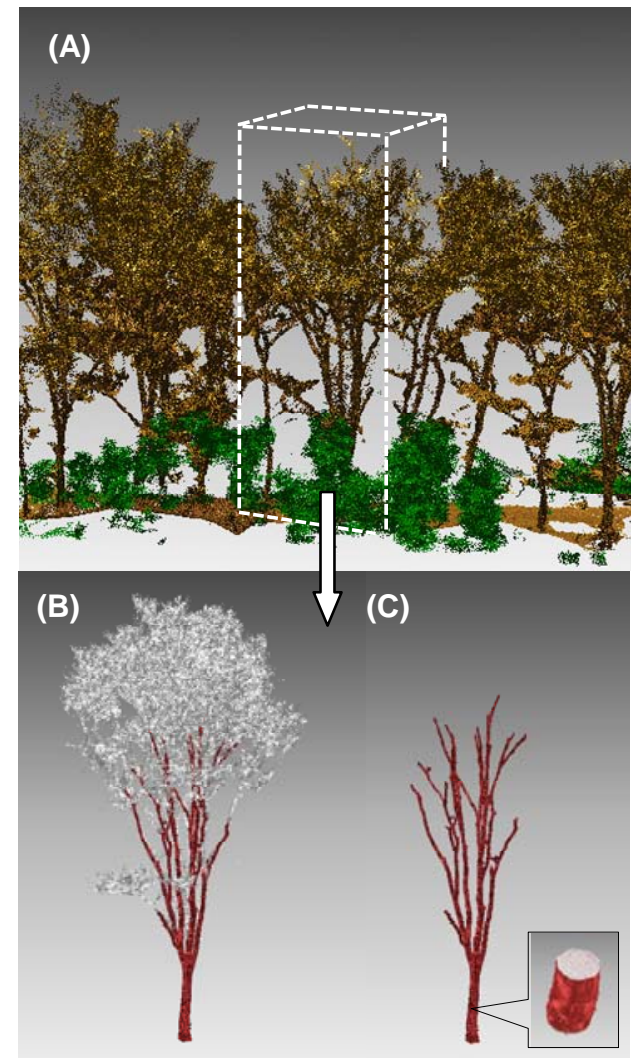

Figure 6: The 3-D voxel-based solid model of the zelkova tree generated from the 3-D point cloud data obtained by portable scanning lidar. (A) A point cloud image of a group of zelkova trees before voxelization. Area enclosed by dashed lines is the target tree within the measurement plot. (B) The 3-D model of the target tree: small branches are white. (C) Stem and large branches only. In the cross-section of a part of the stem, the interior voxels are colored white (modified from Hosoi et al., 2013). 


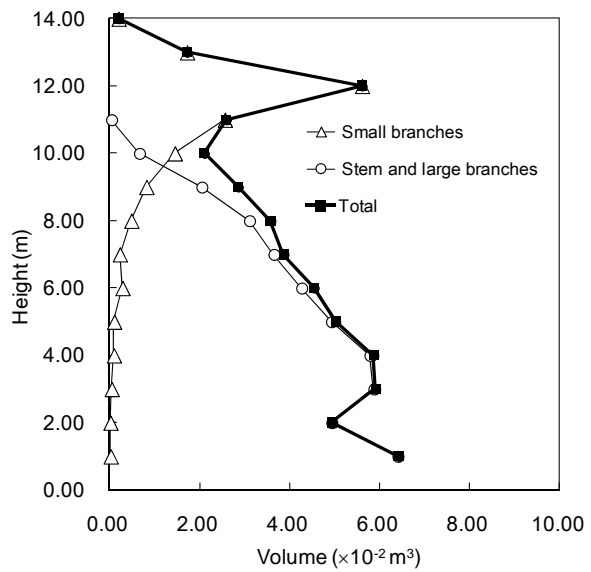

Figure 7: The estimated volume of the stem and large branches, the estimated volume of the small branches, and the estimated total volume, derived from the voxel-based solid model by counting voxels in each $1.0-\mathrm{m}$ height interval (Hosoi et al., 2013).

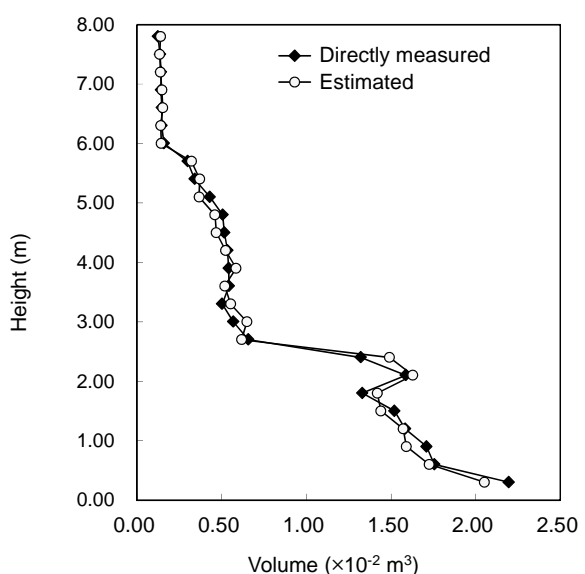

Figure 8: Comparison of the woody material volume of a certain part of the target tree estimated from the voxel solid model and the directly measured volume for each $0.30-\mathrm{m}$ height interval (modified from Hosoi et al., 2013).

\section{CONCLUSIONS}

Accuracy of lidar-derived LAD estimation using the voxel tree model of a broad leaved canopy were improved by combining airborne and portable ground-based lidar data in conjunction with Gaussian interpolation. A proposed laser beam coverage index $\Omega$ could explain the LAD estimation error even in different types of lidars. This index is useful for knowing LAD estimation accuracy without destructive measurements and determining the appropriate laser settings for more accurate LAD estimation. Voxel solid modeling using lidar data could offer accurate estimation of woody material volume. This modeling method utilizes almost all spatial information contained in the lidar-derived 3-D point cloud data to faithfully reproduce the complex shape of the target without any tree shape approximation. Thus, this method is suitable for woody volume estimation of complex shaped trees. Future studies should investigate the applicability of the voxel modeling using lidar data to other regions with different conditions and species, and to different tree structural parameters.

\section{REFERENCES}

Côté, J. F., Fournier, R.A., Egli, R., 2011. An architectural model of trees to estimate forest structural attribute using terrestrial LiDAR. Environmental Modeling \& Software, 26 (6), pp.761-777.

Dassot, M., Constant, T., Fournier, M., 2011. The use of terrestrial LiDAR technology in forest science: application fileds, benefits and challenges. Annals of Forest Science, 68 (5), pp.959-974.

Ghuneim, A.G., 2009. "Moore-Neighbor Tracing Idea". http://www.imageprocessingplace.com/downloads V3/root do wnloads/tutorials/contour_tracing_Abeer_George_Ghuneim $/ \mathrm{mo}$ ore.html (8 June, 2013).

Hosoi, F., Omasa, K., 2006. Voxel-based 3-D modeling of individual trees for estimating leaf area density using highresolution portable scanning lidar. IEEE Transactions on Geoscience and Remote Sensing, 44 (12), pp.3610-3618.

Hosoi, F., Omasa, K., 2007. Factors contributing to accuracy in the estimation of the woody canopy leaf-area-density profile using 3D portable lidar imaging. Journal of Experimental Botany, 58 (12), pp.3464-3473.

Hosoi, F., Nakai, Y., Omasa, K., 2010. Estimation and error analysis of woody canopy leaf area density profiles using 3-D airborne and ground-based scanning lidar remote-sensing techniques. IEEE Transactions on Geoscience and Remote Sensing, 48 (5), pp.2215-2223.

Hosoi, F., Nakai, Y., Omasa, K., 2013. 3-D voxel-based solid modeling of a broad-leaved tree for accurate volume estimation using portable scanning lidar. ISRPS Journal of Photogrammetry and Remote Sensing, 82, pp.41-48.

Hyyppä, J., Kelle, O., Lehikoinen, M., Inkinen, M., 2001. A segmentation-based method to retrieve stem volume estimates from 3-D tree height models produced by laser scanners. IEEE Transactions on Geoscience and Remote Sensing, 39 (5), pp.969-975.

Lefsky, M., McHale, M., 2008. Volume estimates of trees with complex architecture from terrestrial laser scanning. Journal of Applied Remote Sensing 2; doi: 10.1117/1.2939008.

Næsset, E., Gobakken, T., Holmgren, J., Hyyppä, H., Hyyppä, J., Maltamo, M., Nilsson, M., Olsson, H., Persson, A., Söderman, U., 2004. Laser scanning of forest resources: the Nordic experience. Scandinavian Journal of Forest Research, 19 (6), pp.482-499.

Popescu, S.C., Wynne, R.H., Nelson, R.F., 2003. Measuring individual tree crown diameter with lidar and assessing its influence on estimating forest volume and biomass. Canadian Journal of Remote Sensing, 29(5), pp. 564-577.

Schilling, A., Schmidt, A., Maas, H.G., 2012. Tree topology representation from TLS point clouds using depth-first search in voxel space. Photogrammetric Engineering and Remote Sensing, 78(4), pp.383-392. 